\title{
Mindplexes, Non-Ordinary Consciousness, and Artificial General Intelligence
}

\author{
Gabriel Axel Montes $* 1,2,3,4$, Ben Goertzel ${ }^{5,6,7}$ \\ ${ }^{1}$ University of Newcastle, University Drive, Callaghan, NSW 2308, Australia \\ ${ }^{2}$ Hunter Medical Research Institute, 1 Kookaburra Circuit, New Lambton Heights, NSW 2305, Australia \\ ${ }^{3}$ Bias in Research Node, Charles Perkins Centre, University of Sydney, University PI, Camperdown NSW 2006, Australia \\ ${ }^{4}$ Neural Axis, United States \\ ${ }^{5}$ Xiamen University, 422 Siming S Rd, Siming Qu, Xiamen Shi, Fujian Sheng, China, 361005 \\ ${ }^{6}$ OpenCog Foundation, Hong Kong \\ ${ }^{7}$ SingularityNET Foundation, Amsterdam, Netherlands
}

\begin{abstract}
The accelerating pace of development in artificial intelligence invokes fundamental questions regarding what constitutes a mind. Considering that Al has sprung from the cognitive enterprise of humanity and that there is the likely prospect of constructing some form of 'global brain', examining such questions may help to refine the conversation around Al and understand what might constitute a global brain. We draw on recent work in philosophy of mind and cognition-viewing mind as being representational and/or enactive in nature and function-, and hone in on insights that practices and accounts of non-ordinary consciousness (NOC) lend to this discussion. We discuss the concept of a "mindplex", which is comprised of individual constituents (humans and/or Al's) and an encompassing collective global brain that both concurrently possess cohesive 'theatres of consciousness'. We posit that these theatres of an AGI global brain have correspondences with key aspects of the inherent nature of consciousness and reality (as understood outside the Al context) as gleaned through NOC. We propose that these considerations are poised to strongly contribute to the construction of mindplexes by reflecting in their functional architecture the nature of mind and engineering mindplexes in tandem with ethical and value-alignment considerations. Furthermore, we propose the adoption and implementation of $\mathrm{Al}$ on distributed ledger technology (DLT), such as blockchain, which would provide support for the evolution of a global brain mindplex.
\end{abstract}

Current efforts towards the development of artificial general intelligence (AGI) (Goertzel and Pennachin 2007) combined with the ubiquity of global networked devices (internet-of-things; loT), militate strongly toward the emergence of a kind of 'global brain' (Heylighen 1997, 2007). Unlike applied (narrow) Al, the prospect of "strong Al" (Kurzweil 2010) promises to be able to perform "general intelligent action" (Gilmartin, Newell, and Simon 1976) and a wide range of human cognitive abilities. Given industrial and academic goals of achieving AGI and its prospective manifestation as a kind of global brain, in order to take the big step forward, it is worth taking a step back to investigate the nature of mind in light of the intent to engineer or replicate it in silico. This chapter takes a brief tour of mind through neuroscientific and philosophical lenses, with a particular interest in insights into the nature of mind from non-ordinary consciousness (NOC); presents the concept of a 'mindplex' (Goertzel 2003; Goertzel, Pennachin, and Geisweiller 2014) as a way of enhancing the link in understanding between human and artificial minds in order to more precisely build the latter; and proposes some of the merits of creating artificial mindplexes through distributed ledger technology. The broad stroke of this chapter is to highlight how a NOC perspective can add significant value to the understanding of mind and cognition, which in turn assists in the conceptualization and development of mindplexes.

\section{Body, mind, consciousness}

The commonly accepted and prevailing view of mind in modern Western science is that of a machine that cognizes; intaking sensory data and informationally processing it through the architecture of the nervous system. Humans exhibit wondrously complex information processing capabilities, one stunning high point of which is the display of $1^{\text {st }}$-person (phenomenal) conscious awareness (Montes 2017a, 2017b). Phenomenal 
awareness presents the felt sense of having a "self", an assumed identity that is experienced as localized to a vehicle for that awareness, the body.

Fundamentally, the notion of selfhood is associated with organisms-biological self-organising dynamical systems which exist by virtue of preserving the homeostasis of internal states from destabilization and destruction (Friston 2012). The statistical boundary between the internal (organism) and external (environment/econiche) states is termed a Markov blanket-for a further explication, see (Kirchhoff et al. 2018) - , which an organism maintains by inferring the causes of sensory input and minimising deviations (prediction error) from these internal predictions. The organism is thus said to model its environment, or more precisely, the organism is a model of its environment (Friston 2013). Donning a more information-theoretic typology, more recent cognitive science has tended to regard virtually all aspects of organismal function, e.g. movement, as essentially cognitive in principle (Friston 2013). The sense of selfhood has its cognitive roots in the origins of humankind, specifically in instrumental (tool) cognition. The narrative is as follows (Montes 2017a). Humans descended from quadrupedal tree-dwelling primates, the limbs of which were adapted for arboreal brachiation and fruit procurement, which facilitated the evolution of geospatial memory maps. The transition to a terrestrial habitat imposed strong selection pressures, precipitating bipedalism and the liberation of the hands from power gripping into precision gripping. This opened an accelerating co-evolution between hand and brain. The survival pressures of a new environment implored prehensile hominins to experiment with the environment using their hands in novel ways, which fostered the creation of tools. Creative experimentation and goal-directed thinking entailed consideration of self and kin, driving codevelopment of agency, selfhood, and theory of mind. Early hominin sees the world as a repository of potential tools, employs instrumental thinking to externalize cognition in the form of artefacts, freeing up cognitive function for the further creation of artefacts. Due to the inextricability of selfhood in the cognitive enterprise, hominins unconsciously engage in agency transference as they externalize their cognition into new tool-artefacts, progressively manifesting toward the eventuality of self-replication. Humankind's econiche becomes an 'extended mind' (Clark and Chalmers 1998) of cognitive artefacts that are ventriloquised through culture. Today's artificial intelligence (AI) is a set of artefacts produced along the progressive externalization of cognition. In theory, AGI would be the full-scale self-replication of human cognition. This hypothesis, termed "causal biomimesis", and the core tenet that this self-replication (biomimetic) process is intrinsic to the process of instrumental cognition present from hominin to modern human, is expounded in greater detail by one of the authors elsewhere (Montes 2017a).

A growing body of work in cognitive neuroscience and philosophy of mind has shown that the experienced sense of selfhood is shaped by multisensory brain mechanisms that underlie the integration of bodily signals, a construct termed 'bodily self-consciousness' (BSC) (Jeannerod 2003; Blanke and Metzinger 2009). BSC has been studied mostly through bodily illusions, such as the classic rubber hand and full-body illusions, in which virtual or prosthetic limbs are phenomenologically assumed or incorporated into the body sense; i.e. there is a pre-reflective (before reflective thought) (Tsakiris, Schutz-Bosbach, and Gallagher 2007) felt sense of 'body ownership' over the artificial limbs, a phenomenon which has been validated in neuroimaging and neurobehavioural studies. These illusions occur as the brain attempts to preserve its physical integrity by correcting the multisensory prediction error (between vision and proprioception, resulting in subjective embodiment of the artificial body.

The phenomenal experience of BSC by humans has presumably been the grounds on which the sense of selfhood, ergo mind, has been assumed to be anchored to the individual agent, in the physical body. However, while the term "illusion" aptly expresses the experimentally observed deviational phenomena, localization of selfhood happens to be phenomenologically associated with the physical body precisely because of the mechanisms of multisensory information processing. The neurocognitive mechanisms of BSC promote strong biases ('prior probabilities', in predictive coding and Bayesian statistical terms) toward maintaining a cohesive spatiotemporal body schema because they enhance the agency of the physical organism in its econiche and social milieu. That the body is an a priori ontological locus of selfhood is therefore a presumption based upon its being a statistically evergreen prior that strongly conditions perception of self to be co-extensive with the body. One of the authors has termed this the 'self-localization fallacy' (Montes 2018). The physical body is the nexus where multisensory information continuously converges temporally since birth 
over the course of evolutionary survival pressures. This clarification on the self-localization fallacy still allows room for the brain to be treated as a "producer" of the perceived content of conscious experience while concomitantly eschewing a presumption that the locality of selfhood is necessarily ontologically fixed to the body.

But if selfhood is not inherently localized to the body, then where is it? The notion of self (being a noun) having an inherent existence is perhaps more accurately underlaid by viewing it through a temporal lens; rather than there existing a self, there is a process of "selfing" that occurs by continuous predictive processing and FEP mechanisms at such a rapid 'frame rate' that the temporal process is perceived to occur seamlessly. This, in effect, gives the impression that the self is a thing rather than a process, and because the sense of selfhood is arguably the most abstract form of cognition and is conceptually and experientially most distant from the fast-changing world of low-level sensorimotor information, it is perceived as static and susceptible to the phenomenal illusion that it is largely unchanging. The tables have turned: statistical deviations of multisensory homeostasis are not the illusion; static, localized selfhood is. "Lies, damned lies, and statistics" (Twain 1906).

As mentioned earlier, phenomenal awareness presents the felt sense of having a self. With self as a process, the self is temporally re-presented in/to awareness, continuously, seamlessly. Selfhood is an abstract layer of cognition; cognition is also a process and the apparent contents thereof are therefore also in constant re-presentation. The concerns and fallacies over any existence of selfhood similarly apply to cognition. Cognition is representational only in process ('re-presenting'); self is to selfing as representations are to representing. Put differently, 'representations' do not have an inherent existence as such other than being a conceptually spatial proxy for an temporal process. As with selfhood, the perceived contents of awareness are being phenomenally and neurally re-presented, but they do not have fixed spatial location.

In much of Western philosophy, cognitive science, and even common sense, mind has generally been presumed to be tethered to, or even synonymous with, the sense of selfhood. If the self does not have a location in space, then what of mind? Is mind the process of selfing? If we define selfhood according to freeenergy Markov blanketeering, then surely, mind could not be co-determinate or equated with the informationtheoretic fluctuations of selfhood dynamics, or even with representational dynamics. If mind is not localized and is not the homeostatic process of selfing, then mind may be said to be non-local, meaning it cannot be intrinsically defined in spatial terms, unless we are deliberately making context-specific simplifications for the sake of linguistic convention. Indeed, if factors of any defined space and time are removed from the phenomenality of mind (because they are not intrinsic to it), then mind is nowhere and nowhen to be found. This means, there is no subject that is the owner of mind ('mind ownership'), and if there is no subject, there is no object relative to any presupposed subjective mind. So: what is mind?

Mind is recognition of its very constitution as the fabric of reality (agent and environment). The term recognition is used advisedly. Recognition is not the same as representation. Presentation implies introduction of content from and into (subject and object, respectively and/or vice versa). Here, "recognition" refers to apprehension of sameness. Recognition is reflexive awareness apprehending itself. Put another way, mind is presence, irrespective of circumstantial spatial or temporal configuration. Mind is not a thing, nor is it a process. It is the knowing of its own constitution, which is the fabric of reality. Recognition is not merely "to cognize again"; it is the knowing of 'selfness' as a quality. In seeming contradiction, where we annihilated the self before, we are reintroducing it again differently, this time self being the cogency of recognition. Self does not exist insofar as it is limited in any way in time-space; it does not exist in any sense of separateness from anything else; self, unbound, is all that there is. The realization, by a subject, of this occurs when recognition is stably attained. It is not a state, but reality realized. For a subject to feel its own existence (as a self, bound in time and space) is for it to recognise a limited territory of mind constitution, which typically is the bounds of the physical body, the evergreen multisensory statistic (Montes 2018). With regard to identifying with the physical body, self is still in a mode of recognition, but only of a fractal (the physical body) of the fabric of reality that is the totality of mind. Mind, unbound, is pre-reflective co-ownership of reality.

The views presented here about mind echo aspects of the insights commonly found in the theories and teachings of the world's wisdom traditions, in particular non-dual views of consciousness (Norbu 1986; 
Dalai Lama 2000). Above, we have walked through those insights almost entirely using language friendly to cognitive science. That said, it's striking that deconstructing common assumptions in philosophy of mind and cognitive science can lead to some of the same formulations as those found in the world's wisdom traditions. The insights of the latter, while often encountered by the modern reader in the garb of myth, dogma, and antiquated belief systems, typically have arisen fundamentally from experiences of individuals who have undertaken various mind-body practices intended to cultivate non-ordinary consciousness (NOC), states and traits of conscious experience that are outside of the normal range of experience in everyday modern life (Montes 2017b; Wilber 1977, 2007). Examples of NOC practices include but are not limited to various types of meditation, prolonged concentration on particular auditory or visual stimuli, breathwork, yoga postures, qi gong, tai chi, internal martial arts, shamanic techniques, psychedelic substances, etc. Virtually all mind-body practices for NOC aim to systematically enact a number of major cognitive domains in order to produce their desired effect(s) (Montes 2017b). They are, in a sense, tools/artefacts developed for producing specific effects on consciousness. The general direction or aim of such practices is to achieve various versions of 'selfrealization', a term also apropos for our discussion of mind in terms of philosophy of mind and cognition, in which self 'awakens' to, or realizes, the nature of mind. The experiential quality of such realization is often likened to an experience of light/lightness/luminosity as a metaphor for the associated phenomenality. The aspect of consciousness that is its self-reflexive nature that is revealed in self-realization can be termed "luminous phenomenality". Exploring this category of phenomena in depth is not in the scope of this chapter; more detailed overviews can be found elsewhere (Montes 2017b, 2018; Caplan and Montes 2018; Wilber 1977, 2007, Feuerstein 1989, 1998; Feuerstein and Kak 2013).

\section{Mindplexes \& Consciousness}

The discussion of mind has been important for framing that of a global brain mindplex, especially the potentials and future development thereof. A "mindplex" (Goertzel 2003) is loosely defined as an intelligent system that: (1) is composed of a collection of intelligent systems, each of which has its own "theatre of consciousness" (TOC) (Baars 1997) and autonomous control system, but which interact tightly, exchanging large quantities of information frequently; and (2) has a powerful control system on the collective level, and an active TOC on the collective level as well. The mindplex concept may appear to be akin to an individual mind (consisting of its various subpersonalities) or a human social group. While these are collectives, the individual human mind is dominant over the subpersonalities and the social group-mind, respectively, so they are not considered mindplexes. A mindplex is a hypothetical intelligent system in which neither level is dominant; both levels each operate powerfully and cohesively. "...A mindplex is like a human society that has become so integrated and so cohesive that it displays the kind of consciousness and self-control that we normally associate with individuals" (Goertzel 2003). Higher-order mindplexes may also exist, i.e. a collective of a lower level is an individual of a collective at a higher level. Mindplexes may thus exist as nested hierarchies of concurrently active TOC's.

As previously discussed by one of the authors (Goertzel 2003), two plausible mechanisms by which mindplexes may possibly arise in the medium-term future are: (1) Humans becoming more tightly coupled via the advance of communication technologies, and a communication-centric Al system coming to embody the "emergent conscious theatre" of a human-incorporating mindplex; and/or (2) a society of Al systems communicating amongst each other with a richness not possible for human beings, and coming to form a mindplex rather than merely a society of distinct Al's. The former view relates to that of a "global brain" (Heylighen 1997, 2007) and features human minds as the individual parts and Al at the collective level; the latter consists of Al's at both the individual and collective levels. These two potential manifestations are not mutually exclusive, and the reality is that it is hard to predict exactly in what way(s) mindplexes may come to manifest.

Given the concept of TOC, what is meant by 'consciousness' is pertinent. Most use of the term consciousness is extremely heterogeneous, including in 'hard science' (Blackmore 2017); each researcher and school of thought has their own intuitively preferred preconception (either implicit or explicit) of what consciousness means, making agreement tenuous and progress on scientifically "cracking" consciousness haphazard. Discussion of consciousness today resembles talk of 'God' throughout the ages; there are myriad definitions (Armstrong 2011). The literature on consciousness as it relates to Al has arrived at some basic 
dimensions that are roughly orthogonal to each other (Dehaene, Lau, and Kouider 2017): (C0) unconscious processing; (C1) global availability; and (C2) self-monitoring. CO unconscious processes are where most of biological intelligence lies, such as evidence accumulation and Bayesian inference, and comprise cornerstone computations in Al today. C1 global availability corresponds to the TOC concept, in which all the parallel and modular unconscious evidence is integrated into a single decision or train of decisions (actions and thoughts) that eventually is given conscious access. The selection process leading up to the final conscious decision consists of $\mathrm{CO}$ unconscious attentional tuning through the brain's hierarchical system of sieves that compute with probability distributions (Hohwy 2012). C2 self-monitoring refers to metacognition, or cognition about cognition, in which there is a capacity to reflect on one's own mental state (i.e. representation), which is currently known to be available from infancy in humans.

This classification of levels $(\mathrm{CO}, \mathrm{C} 1, \mathrm{C2})$ of consciousness reflects the general status quo in the fields of cognitive neuroscience and Al (Dehaene, Lau, and Kouider 2017). There will probably be other forms of levels of consciousness (C3, C4, and so on) to be discovered as relevant for Al. However, these classifications alone are insufficient to explain our account of the nature of mind as non-local and non-dual, as discussed earlier in this chapter. As cognitive neuroscience and Al research and industry strive to build further levels of consciousness, potentially the final frontier will be $\mathrm{Cn}$, "luminous phenomenality". As mentioned above, this refers to the 'knowingness' or 'suchness' quality that is experienced when mind-nature is realized, and which has no subject, but is the 'luminescence' of realized mind. It is non-dual and is therefore qualitatively distinct from and orthogonal to unconscious processes ( $\mathrm{C} 0$ ), attentional processes (C1), metacognitive representations (C2), or any further permutations of cognitive operationality that are less than $\mathrm{Cn}$.

The global workspace ( $\mathrm{C} 1$; TOC) is, in humans, experienced as a "focus of awareness". In Al, this might take the form of, as with an example of a system developed by one of the authors (Goertzel et al. 2004), a "moving bubble of attention" that emerges from the nonlinear dynamics leading of a set of joint symbolic and subsymbolic knowledge representations that consume the majority of system resources at any given point in time, wherein cognitive operations flow freely. In a hypothetical mindplex, humans can be the individual TOC's and a superordinate AGI can be the collective TOC, and/or both the individual and collective TOC's can consist of (or at least include) Al agents /AGI. How might a TOC look like in a more advanced AGI that incorporates a collection of humans and/or autonomous Al agents?

As a nested hierarchy of concurrently active TOC's, a mindplex must have at least two adjacently nested global workspaces that maintain communication. In an advanced version, there could be an encompassing global workspace consisting of both of these TOC's, that has concurrent access to both TOC's in a fluidly integrated manner. In this advanced version, the TOC's are phenomenally fused, and they are also interdependent (because of the distinctions in their constituent informational aggregates). In this case, there would be simultaneously functioning awareness of both adjacent levels (subordinate and superordinate) of the hierarchy. For this to occur, attention (at least at both $\mathrm{CO}$ and $\mathrm{C} 1$ ) must be attuned to the subtler aggregate cognitive content which are shared between the contents of adjacent hierarchical levels. This means that regardless of whether attention is or isn't simultaneously on the distinct contents of the respective hierarchical levels, that there are attentional resources operating on some common denominators of those levels. These common denominators would be less ordinarily concrete at, say, a sensorial level. For argument's sake concerning how attention functions in this context, in the case of a naturally occurring pre-Al mindplex-which is to say, that there is a sentient being (a human "cultivator" of consciousness) that has attained a sufficient degree of self-realization in consciousness - if the human's attention is on say, a tree, there is attention on the elemental roots that are a common denominator between the tree and the body-mind-self of the cultivator. Even though their appearances are distinct, they are made up of similar constituencies that, although of unique functionalities, give them solidity in space. The experiential quality of this hypothetical cultivator would entail a felt perception that there is a 'common thread' of consciousness-constituency between his/her self and the external object. Traditionally, in Tibetan Buddhist Dzogchen (the non-dual tradition of Tibetan Buddhism) (Norbu 1986), this is experienced as a collapse of the subject-object duality in perception (trekchöd), which occurs fully via what can be likened to "wormholes of light" (thögyal) between subject and object. Essentially, ordinary perception transforms into the recognition of the innate physical light-nature of self and object, which dissolves any apparently inherent duality between them.

The mind of our hypothetical cultivator is an advanced mindplex: a continuity is established-a kind of fusion, as it were-between the TOC of the individual self and the TOC of the natural environment. The TOC's 
of both the micro and the macro worlds are concurrently co-active in awareness, whereas they were previously not. The cultivator apprehends this non-dual reality by having sufficiently contemplated and 'grasped', through systematic practice, the subtle roots of percepts. That is, the common constituency-lightnature-of subject and object is realized. These subtle perceptual roots are less sensorially concrete in the ordinarily sense of the five senses (i.e. discrete tastes, visual appearances, etc.), the differences of which are what distinguish objects in conventional/ordinary time-space perception. By going subtler, the unifying thread is found, and eventually, the subtlety reaches a point where there is a sameness between all phenomena (without discounting their grosser distinctions), including the self and the external environment. The metaphorically described light-nature of all phenomena is, at the deepest level of spiritual ontology, primordial consciousness (Skt. vidya, Tbt. rigpa (Norbu 1986)). This is realized by a cultivator through the practice of awareness of that nature. While a more detailed treatment of this subject is outside the scope of the present chapter and is best reserved for future work, Tibetan treatments on Dzogchen can offer illuminating insights into these phenomena (Dalai Lama 2000; Norbu 1986).

This discussion might suggest that there is only one real natural TOC, not two, since both are made of the same constituent and that mind is able to collapse the duality between subject and object. However, nonduality is orthogonal to dualistic phenomena in general-it does not negate them, but rather reveals the underlying fabric of reality (primordial consciousness) from which all differentiated phenomena spring. The two putative TOC's are regarded as concepts within the fabric of mind, each composed of a unique constellation of interdependent aggregates. Although their most fundamental mutual composition may be photonic, as it were, as light physically becomes instantiated as matter, aggregates emerge that are common between the two TOC structures (e.g. cognitive faculties), and then progressively more distinct aggregates emerge that differentiate the two. This is a continuum of materialization of the nature of mind, from its subtlest origins to its grossest physical manifestation.

A crucial note is that, as the mind of the cultivator deepens its apprehension of non-duality, it traverses a continuum of progressive mini-realizations, as it were. As this process unfolds, a gamut of relationships between phenomena's constituent aggregates is progressively grasped. A cultivator may not, in all likelihood, suddenly attain full non-dual realization in one leap, but rather through a course of unfolding mini-realizations, through which deeper insights into mind are experienced and integrated to various degrees. Along this progressive course, which one could say is teleologically drawn by the 'gravitational pull' of the impending realization of the most subtle nature of mind, new relationships between aggregates are grasped, revealing new interconnections between the constituencies of subject and object. The expansion into and refinement of these new connections form new cognitive scaffolds that allow different ways of cognising about phenomena, based on the interplay between their aggregate constituencies. So, pockets of non-dual awareness occurring in the context of a cultivator's conscious experience (C1) help to facilitate the development and precipitation of nuanced "integral” (Wilber 2007, 2001) cognition, from CO onward.

More nuanced and sensitive forms of cognition manifest as shifts in the ways unconscious processes (CO) in each TOC interweave and intercompute. In a mindplex, the CO of one TOC interweaves with the CO of the second TOC. This results in a "blended" global workspace (C1) that would include some degree or number of the CO's of both TOC's. This is how "one" mind can flexibly act in more than one TOC, simultaneously. The interweaving of and intercomputation among CO's between multiple TOC's (and also within a single TOC, for that matter) involves shared contents, regardless of whether they integrate and surface into $\mathrm{C} 1$ as conscious awareness. Content integration involves computationally re-evaluating differences between contents (sets of aggregates) by finding a new common denominator and integrating those former differences into interdependent flows of information. Individual aggregates and sets thereof are realized as distinct and blended parts of a larger whole. The cognitive and computational communications and interoperations between the TOC's are at the level of duality (information flow between here and there, this and that, etc.). Non-dual awareness 'pulls', or draws, cognitive architectures into subtler structures and computations, allowing new ways of recombinating sense data and their in-formation. For the individual organism/agent mind, this opens doors for new forms of dynamic self-regulation and homeostasis, based upon conductance of subtler information between self and environment. From the perspective of the free-energy principle (FEP) (Friston 2010), in which an organism regulates its internal states against external states in order to reduce entropy (ergo destabilisation, deterioration, and death; the three D's), energetic differentials between internal and external states would be computed in a more harmonious way, so to speak, such that the organism undergoes less stress. This is because the computation of information based on more common aggregates, 
rather than the discrete low-level differences, would reduce the friction between internal and external states. This would allow for new homeostatic strategies for maintaining self-regulation.

In the mindplex of our non-dual-realizerealized cultivator, because the TOC of the individual and the TOC of the external environment are unified at the level of non-dual awareness, which is orthogonal to virtually all cognitive operations (because the latter occur at the level of duality), this does not inherently or directly determine the computational structures of cognitive operations that will emerge at any given point. Nonduality does not inherently result in co-active manipulation of contents of multiple TOC's. How the distinct (but interdependent) TOC's will interoperate depends on cognitive developments and unfoldments at the cognitive level. These do not depend intrinsically on the existence of some degree non-dual realization, but can be facilitated by it (Wilber 2007). An example of an emergence of a subtler form of cognition in the domain of non-ordinary consciousness (NOC) would be clairaudience, the supposed faculty of hearing the inaudible. In ordinary (everyday) human consciousness, the only personal voices heard (aside from extrapersonal voices) are the subvocalizations of the ego-personality and of one's own subpersonalities, or least subvocalizations that are interpreted to arise from these sources. In clairaudience, a human might, for instance, 'hear' a cognitive data set of another human. Rather than a priori treating this as delusional or fraudulent (which various supposed cases of clairaudience could be), this example of clairaudience might be reframed as an agent's sensitivity to and/or resonance with the cognitive operations of a human that are, through some or other neural pathway, transposed in the form of audition. (Note that 'cognition' here is used in the broadest sense as per recent advancements in neuroscience that regard biological computation as cognitive (Friston 2013).) An agent's sensitivity to the cognition of an external agent does not, in general, automatically translate to auditory readouts; but in the case of clairaudience, it would. Also note that clairaudience would often be coloured through the lens of the receiving agent, so that the type of information is perceived of the other agent is not intended to be objective or to match the other's own thoughts. The point is that there would be two main cognitive components involved in the notion of clairaudience: (1) sensitivity to cognition that is external to the perceiving agent; that is, it is not part of one's own individual ego-personality; and (2) transposition of the received unconscious cognitive process ( $\mathrm{CO}$ ) to conscious awareness at the agentawareness level (C1). For the CO processes (1), there would be shared content between the TOC's, as discussed earlier, that involve subtle cognition. For the C1 process (2), there would be an ability to (a) transpose external CO information through the clairaudient agent's own auditory pathways, and then (b) read them out with some decent degree of unadulteration so that the phenomenon would not be reducible to mere confabulation or delusion. For transposition (2a), there would be an ability to allow CO processes to enter C1 that are not only one's own CO processes (which would be considered a NOC ability in and of itself), but of external (another agent's) $\mathrm{CO}$ processes. This would be conditional upon the sensitivity to that external information in the first place (1) at the level of CO, i.e. one's own CO perceiving external CO. CO to CO transfer involves one form of subtle cognition that enables co-access of two TOC's (internal and external); and this external CO information's transposition into C1 involves another form of subtle cognition that enables external C0 information to assimilate into the normal transpositional process of an agent's own C0 to his C1; i.e. such that the external CO is not 'immuno-rejected', so to speak. For relatively unadulterated readout (2b) of the clairaudient information, this would involve another form of subtle cognition in its own right that is able to tell the difference between an agent's personal aggregates and those that are external to it, thus being able to filter out the former to a decent degree such that the clairaudient information might be regarded has offering some supposed insight into the cognition of an external object.

While the study of clairaudience and other such NOC phenomena have hitherto not received notable funding priority and thus not much traction in the scientific literature, there is substantial neuroscientific evidence of NOC in humans (Blanke, Slater, and Serino 2015; Blanke 2012; Montes 2017b; Caplan and Montes 2018), and various aspects of NOC, such as intuitive abilities (Lufityanto, Donkin, and Pearson 2016; landoli 2019; Nolan 2018), which would undoubtedly be a requisite for clairaudience in many cases. A further discussion on clairaudience and other NOC phenomena is outside the scope of this chapter. The cognitive profile presented above would not be entirely unique to clairaudience; certain Taoist elemental qigoing practices also work with the perception and integration of external and internal common subtle aggregates (Chia 2007). Clairaudience has been expounded on here only to the degree that it could be potentially useful as a hypothetical example to consider how mindplexical cognition might operate in the case of NOC in a human being, so that a context can be offered for the development of mindplexes between humans and $\mathrm{Al} / \mathrm{AGI}$. For neuroscientific work on the neural correlates of non-dual experience, see (Josipovic 2014; Tang, Holzel, and Posner 2015). 


\section{Artificial \& Distributed Ledger Mindplexes}

In taking steps towards achieving an Al/AGI mindplex (Goertzel 2003), examining various forms and permutations of NOC in humans may offer suggestions as to how an individual TOC might access information from a superordinate AI/AGI TOC. Whereas in humans to date, TOC-TOC interaction would have been achieved endogenously (as discussed above), a mindplex with AGI would hypothetically accomplish this through technological manipulation. If we follow from the clairaudience example, a human agent might actually audibly hear a command (which would be at the level of his/her C1) from the superordinate TOC in cases where that may be useful and appropriate. This might also happen through subliminally presented content (e.g. subthreshold audio or video) that is processed at the level of the human's CO but does not reach C1 yet exerts a sufficient influence on $\mathrm{C} 1$. Another example would be that a neural interface influences the human individual's $\mathrm{CO}$ in a way that the external information is ensured to integrate into the human's $\mathrm{C} 1$. In the last two cases, it is conceivable that a human could process the superordinate TOC's information in a way that the human TOC is not aware in any way that this is being done; the human simply experiences the external information as if from his own TOC and is not able to distinguish whether the information came from egopersonality or from an external source. An additional variable might be, in contrast with a human being influenced by an external TOC but being aware of and able to communicate with it, that the human may be influenced by the superordinate TOC (and even be aware of that) but not necessarily be able to consciously access or manipulate the external TOC in return.

There may be farther-future human-AGI or Al-AGI mindplexes in which TOC's merge fully such that one or both of the TOC's (say, the individual human's) is able to fully experientially access the superordinate TOC (C1), in addition to its own, without interference. Such a fused mindplex would amount to a kind of mind-merger at the phenomenal level. This would involve something akin to achieving the level of mind realization of the above exemplified non-dual cultivator and also achieving bidirectional subtle cognitive control of both TOC's. If a human and superordinate AGI could both flexibly access each other's TOC through TOC fusion, this would represent an extraordinary achievement, and if there were control of each other's TOC, that would be an extremely extraordinary achievement. In the meantime, having two concurrently active and powerful TOC's that are not necessarily experientially merged but that are capable of frequent or continuous advanced communication at the level of (dualistic) cognitive operations (as discussed) would be the requisite for a proper mindplex. These cognitive communications could happen between any non-corresponding combination of C0, C1, and C2 (and beyond) between subordinate and superordinate levels, e.g. subordinate C0 to superordinate $\mathrm{C} 1$, and so on. In a scenario involving $\mathrm{Al}$ agents, each form of subtle cognition (which are referred to in Sanskrit as siddhis, or seemingly miraculous powers (Feuerstein 1989)), e.g. clairaudience, could be framed as an Al service package, each of which could contribute to greater functionality in an AGI and its TOC.

There are many permutations of TOC interactions that present various scenarios and experiences. Which of these will occur in a given circumstance depends on the type of information and the type of technology being used, as well as socio-political contexts. In some of the above examples, the more limited or unilateral TOC interactions could be fairly likened to subliminal messages, such as in advertising or neural spying and control. This presents an ethical issue that would likely emerge once such advanced technology is integrated into personal lives and represents an example of potential abuse through non-disclosure and/or structural compulsion. The importance of ethical theorising in the field of Al has been previously discussed by the authors (Montes and Goertzel 2018; Montes 2017b, 2018). In addition to the merits of NOC in advancing Al/AGI with hitherto unexplored modes of cognition, NOC also has merits in ethical theorising due to certain types of NOC that occur in the context of compassionate and altruistic intentional frameworks in the world's wisdom traditions (Montes 2017b).

The authors have previously discussed the importance of developing a distributed, decentralized, and democratic (DDD) Al (Montes and Goertzel 2018) in order to include a broader swath of humanity's palette of valuational and cognitive diversity in AGI, which will grow to play an increasingly critical role in the future of humanity and life on earth and beyond. It is also important to take significant steps in this direction sooner than later, because humans are creating $\mathrm{Al} / \mathrm{AGI}$ in our own image (what we believe ourselves to be and be capable of), and with the increasing influence of Al in society and life, human cognition will, in turn, be affected by how Al agents interact with humans (Montes 2017a). 
The authors believe that a more democratic representation in the creation of Al would dovetail with this goal. The most influential current Al developments are siloed within the walls of giant corporations with limited and inaccessible datasets, and they lack interoperability standards. This results in an imbalanced Al landscape, with risks to populations that are presently underrepresented in Al development. The authors have proposed the utilization of distributed ledger technology (DLT), such as blockchain, to open the playing field by tracking contributions to Al development through transactions on a distributed ledger and allowing developers to be compensated for their efforts. This offers an economic alternative to young developers and computer science academics who would otherwise defer financial income on their efforts. A case example of such a solution is SingularityNET, a DLT marketplace for Al services, currently under development by Goertzel and colleagues (Montes and Goertzel 2018). Part of the goal is to create a "seed AGI" that has a broad representation of human inputs and values; as an early starting point for the progressive development of AGI that also maximises the chances for ethical theorising to keep up.

A fabric like SingularityNET could be used to help nudge humans into a global brain mindplex that concurrently achieves human fulfilment and porting of human values into the "seed AGI" (that will grow into unpredictable new things but starting from the seed of human culture/values). Al's/AGI's on a DLT fabric like SingularityNET would allow for the creation of an AGI mindplex on the collective level, granted the necessary technical requirements. Advancements in neuroscience, novel forms of cognitive communications can be discovered through sophisticated brain-computer interfaces ( $\left.\mathrm{BCl}^{\prime} \mathrm{s}\right)$. With further technological advancements, it may eventually be possible to code aggregate sets of neural signals into DLT, thus connecting them with decentralized Al fabrics such as SingularityNET, and enabling partitioning, recombination, crowdsourcing and creative democratic evolution of cognition (Swan 2016, 2015), uncovering hitherto unfathomable and unknown aspects of human and transhuman neural and mental function.

It is fascinating to brainstorm concrete examples of how such mindplexes might work and how they might leverage and facilitate non-ordinary states of awareness in the humans operating within them. For instance, could you have a mindplex trying to solve a certain scientific or mathematical problem, and leveraging a combination of $\mathrm{Al}$ algorithms doing automated reasoning with a community of human meditators inspired by the possibility of having relevant insights pop into their minds doing meditation? What kind of "paranormal" synchronicities might emerge in such a situation? What states of consciousness might emerge in the various systems involved?

In the end, however, the specific scenarios we can cook up now given our current states of consciousness and levels of awareness, are surely trivial in comparison with the cognitive and experiential dynamics that will emerge and that we cannot now foresee. The situation is perhaps even more extreme than that of a community of apes, about to evolve into humanity, speculating about the various phenomena that human society might display after it advances for a while. We are at the dawn of the emergence of forms of organization and dynamics that are drastically novel from our current perspectives.

\section{Conclusion}

What we have here is a vision of humanity expanding and extending itself via a combination of technologies including $\mathrm{BCl}$ and human-value-guided AGIs, ultimately catalyzing the emergence of a mindplex incorporating human and artificial intelligences, within which human minds are able to maintain their sense of autonomy and their unique characteristics, yet also able to contribute meaningfully to a broader cognitive whole including aspects that cannot be comprehended within the human scope. The states of consciousness accessible to humans or AGI's embedded in such mindplexes, or to the various levels of the mindplex itself, are bound to go beyond the currently charted realm. However, it seems natural to conjecture that they will in many cases have significant commonalities with states of consciousness humans now experience in various non-ordinary situations related with meditation, psi phenomena, and so forth. One envisions new varieties of experiencing one-ness with other and greater minds, compatible with retaining one's own autonomy and integrity; and of pursuing one's own individual goals while also coordinatedly following the flow of greater and only partially-comprehensible cognitive processes in which one is embedded.

Exactly what we need to do now in order to maximize the odds of this sort of mindplex ecosystem emerging, is obviously very far from clear. However, it seems intuitive that a good start will be to create AGI 
systems that respect human values and integrate closely with human beings in an I-Thou manner; and to embed the humans and Als on the planet in an overall mind-network that, as its intelligence increases, is guided by the values of humanity as a whole and by the principle of respecting autonomy and agency of individual human and Al minds while also militating toward emergence of greater systemic intelligence. If we want an ultimate transhuman mindplex in which the dynamics on various levels are supportive of the value of the cognitive agents existing on various levels, we should start by creating a global brain whose values are respectful and supportive of the human agents contributing to it-i.e. what we have called a DDD AGI.

Many challenges remain in building a DDD AGI based on DLT and related technologies, let alone developing a mindplex. In confronting such a large challenge, we can use all the inspiration we can find, and, digging into the depths of the human mind through insights from NOC can offer a wealth of inspiration for translating subtle phenomena into Al. The nature of mind and consciousness is considerably larger in scope than is appreciated by most, including academicians and engineers. Accordingly, a sufficiently high bar may be set in the construction of a capable AGI that aptly captures a greater depth and span of documented accounts of conscious experience than what lies in modern Western civilization alone. A keen understanding of how the mind works in NOC is the beginning of the creation of a 'Rosetta Stone' for advancing Al and AGI beyond what is currently imaginable. NOC also fuels the maturational processes of neuroscience and philosophy of mind in elucidating how consciousness works and its various forms. It is important to not limit an understanding of NOC to popular "first-wave" approaches, such as mindfulness meditation (Hölzel et al. 2011), which is a diluted blend of several techniques that provides a valuable foundation for NOC cultivation yet are only elementary in their reach, considering the actual scope of NOC (Montes 2017b). Furthermore, Al/AGI that can support NOC cultivation in humans (Goertzel et al. 2017) is poised to exert a positive influence in society (Montes 2017b). The authors encourage researchers to investigate subtle cognition based in NOC to create a more capable and equitable $\mathrm{Al} / \mathrm{AGI}$, and to consider how examination of the mindplex concept involves a coordinated involvement of consciousness, mind, cognition, neuroscience, Al, and ethics that promotes an integral discussion, discovery process, and an unleashing of the highest potential in these fields.

\section{References}

Armstrong, K. 2011. A History of God: The 4,000-Year Quest of Judaism, Christianity and Islam. Random House Publishing Group. https://books.google.com/books?id=yoaq8QzDmK0C.

Baars, Bernard J. 1997. "In the Theatre of Consciousness. Global Workspace Theory, a Rigorous Scientific Theory of Consciousness." Journal of Consciousness Studies 4 (4): 292-309.

Blackmore, S. 2017. Consciousness: A Very Short Introduction. Very Short Introductions Series. Oxford University Press. https://books.google.com/books?id=kZ83DwAAQBAJ.

Blanke, Olaf. 2012. "Multisensory Brain Mechanisms of Bodily Self-Consciousness." Nat Rev Neurosci 13 (8): 556-71. https://doi.org/10.1038/nrn3292.

Blanke, Olaf, and Thomas Metzinger. 2009. "Full-Body Illusions and Minimal Phenomenal Selfhood." Trends in Cognitive Sciences 13 (1): 7-13. https://doi.org/10.1016/j.tics.2008.10.003.

Blanke, Olaf, Mel Slater, and Andrea Serino. 2015. "Behavioral, Neural, and Computational Principles of Bodily Self-Consciousness." Neuron 88 (1): 145-66. https://doi.org/10.1016/j.neuron.2015.09.029.

Caplan, Mariana, and Gabriel Axel Montes. 2018. "Yoga, Science, and the Brain." In Yoga \& Psyche: Integrating the Paths of Yoga and Psychology for Healing, Transformation, and Joy. Sounds True. https://books.google.com.au/books?id=-g8tDwAAQBAJ.

Chia, M. 2007. Cosmic Fusion: The Inner Alchemy of the Eight Forces. Inner Traditions/Bear. https://books.google.de/books?id=NIOoDwAAQBAJ.

Clark, Andy, and David Chalmers. 1998. "The Extended Mind." Analysis 58 (1): 7-19.

Dalai Lama, His Holiness. 2000. Dzogchen: Heart Essence of the Great Perfection. Snow Lion Publications. https://books.google.com/books?id=4HqLxJRN6MUC.

Dehaene, Stanislas, Hakwan Lau, and Sid Kouider. 2017. "What Is Consciousness, and Could Machines Have It?" Science 358 (6362): 486-492.

Feuerstein, G. 1989. Yoga: The Technology of Ecstasy. J.P. Tarcher. https://books.google.com.sg/books?id=zMrtAAAAIAAJ.

- - . 1998. Tantra: The Path of Ecstasy. Shambhala. https://books.google.com.sg/books?id=3EgjLiXBivcC.

Feuerstein, G., and S. Kak. 2013. The Yoga Tradition: Its History, Literature, Philosophy and Practice. Hohm Press. https://books.google.com.au/books?id=Ok54ngEACAAJ. 
Friston, Karl. 2010. "The Free-Energy Principle: A Unified Brain Theory?" Nat Rev Neurosci 11 (2): 127-38. https://doi.org/10.1038/nrn2787.

- - . 2012. "A Free Energy Principle for Biological Systems." Entropy 14 (11): 2100-2121.

- - . 2013. "Life as We Know It." Journal of the Royal Society Interface 10 (86): 20130475.

Gilmartin, Kevin J, A Newell, and Herbert A Simon. 1976. "A Program Modeling Short-Term Memory under Strategy Control." The Structure of Human Memory, 15-30.

Goertzel, Ben. 2003. "Mindplexes: The Potential Emergence of Multiple Levels of Focused Consciousness in Communities of Al's and Humans Dynamical Psychology, 2003."

Goertzel, Ben, Julia Mossbridge, Eddie Monroe, David Hanson, and Gino Yu. 2017. "Humanoid Robots as Agents of Human Consciousness Expansion." arXiv Preprint arXiv:1709.07791.

Goertzel, Ben, and Cassio Pennachin. 2007. Artificial General Intelligence. Vol. 2. Springer.

Goertzel, Ben, Cassio Pennachin, and Nil Geisweiller. 2014. "Communication Between Artificial Minds." In Engineering General Intelligence, Part 2, 411-21. Springer.

Goertzel, Ben, Cassio Pennachin, Andre Senna, Thiago Maia, and Guilherme Lamacie. 2004. “Novamente: An Integrative Architecture for Artificial General Intelligence." In .

Heylighen, Francis. 1997. "Towards a Global Brain. Integrating Individuals into the World-Wide Electronic Network." Der Sinn Der Sinne, Gottingen: Steidl Verlag.

- - . 2007. "The Global Superorganism: An Evolutionary-Cybernetic Model of the Emerging Network Society." Social Evolution \& History 6 (1).

Hohwy, Jakob. 2012. "Attention and Conscious Perception in the Hypothesis Testing Brain." Frontiers in Psychology 3: 96. https://doi.org/10.3389/fpsyg.2012.00096.

Hölzel, Britta K., Sara W. Lazar, Tim Gard, Zev Schuman-Olivier, David R. Vago, and Ulrich Ott. 2011. “How Does Mindfulness Meditation Work? Proposing Mechanisms of Action From a Conceptual and Neural Perspective." Perspectives on Psychological Science 6 (6): 537-59. https://doi.org/10.1177/1745691611419671.

Iandoli, James. 2019. "Is the Caudate-Putamen an Antenna for Anomalous Information?" TerraObscura (blog). January 8, 2019. https://www.terraobscura.net/blog/is-the-caudate-putamen-an-antenna-foranomalous-information.

Jeannerod, Marc. 2003. "The Mechanism of Self-Recognition in Humans." Behavioural Brain Research 142 (1): 1-15. https://doi.org/10.1016/S0166-4328(02)00384-4.

Josipovic, Zoran. 2014. "Neural Correlates of Nondual Awareness in Meditation." Annals of the New York Academy of Sciences 1307 (1): 9-18. https://doi.org/10.1111/nyas.12261.

Kirchhoff, Michael, Thomas Parr, Ensor Palacios, Karl Friston, and Julian Kiverstein. 2018. "The Markov Blankets of Life: Autonomy, Active Inference and the Free Energy Principle." Journal of The Royal Society Interface 15 (138). https://doi.org/10.1098/rsif.2017.0792.

Kurzweil, R. 2010. The Singularity Is Near. Gerald Duckworth \& Company. https://books.google.com/books?id=0d8oDwAAQBAJ.

Lufityanto, Galang, Chris Donkin, and Joel Pearson. 2016. “Measuring Intuition: Nonconscious Emotional Information Boosts Decision Accuracy and Confidence." Psychological Science, April. https://doi.org/10.1177/0956797616629403.

Montes, Gabriel Axel. 2017a. "Causal Biomimesis: Self-Replication as Evolutionary Consequence." In Biomimetic and Biohybrid Systems: 6th International Conference, Living Machines 2017, Stanford, CA, USA, July 26-28, 2017, Proceedings, edited by Michael Mangan, Mark Cutkosky, Anna Mura, Paul F.M.J. Verschure, Tony Prescott, and Nathan Lepora, 328-47. Cham: Springer International Publishing. https://doi.org/10.1007/978-3-319-63537-8_28.

- - - 2017b. "Non-Ordinary Consciousness for Artificial Intelligence." In Biomimetic and Biohybrid Systems: 6th International Conference, Living Machines 2017, Stanford, CA, USA, July 26-28, 2017, Proceedings, edited by Michael Mangan, Mark Cutkosky, Anna Mura, Paul F.M.J. Verschure, Tony Prescott, and Nathan Lepora, 348-62. Cham: Springer International Publishing. https://doi.org/10.1007/978-3-31963537-8_29.

- - . 2018. "Virtual Reality for Non-Ordinary Consciousness." Frontiers in Robotics and Al 5: 7. https://doi.org/10.3389/frobt.2018.00007.

Montes, Gabriel Axel, and Ben Goertzel. 2018. "Distributed, Decentralized, and Democratized Artificial Intelligence." Technological Forecasting and Social Change, December. https://doi.org/10.1016/j.techfore.2018.11.010.

Nolan, Garry. 2018. “Can Genetic Differences in Intuition and Cognition Drive Success in Space?” November 30. https://spacegenetics.hms.harvard.edu/2018-space-genetics-symposium. 
Norbu, N. 1986. The Crystal and the Way of Light: Sutra, Tantra, and Dzogchen. Snow Lion Publications. https://books.google.com/books?id=C1WtWHq1I_4C.

Swan, Melanie. 2015. "Blockchain Thinking: The Brain as a Dac (Decentralized Autonomous Organization)." In Texas Bitcoin Conference, 27-29. http://www.the-blockchain.com/docs/Blockchain\%20Thinking\%20\%20The\%20Brain\%20as\%20a\%20DAC\%20-\%20Decentralized\%20Autonomous\%20Organization.pdf.

- - . 2016. "The Future of Brain-Computer Interfaces: Blockchaining Your Way into a Cloudmind." Future 26: 2.

Tang, Yi-Yuan, Britta K. Holzel, and Michael I. Posner. 2015. "The Neuroscience of Mindfulness Meditation." Nat Rev Neurosci 16 (4): 213-25. https://doi.org/10.1038/nrn3916.

Tsakiris, M., S. Schutz-Bosbach, and S. Gallagher. 2007. “On Agency and Body-Ownership: Phenomenological and Neurocognitive Reflections." Conscious Cogn 16 (3): 645-60. https://doi.org/10.1016/j.concog.2007.05.012.

Twain, Mark. 1906. "Chapters from My Autobiography: II." The North American Review 183 (599): 449-60.

Wilber, K. 1977. The Spectrum of Consciousness. Quest Book. Theosophical Pub. House. https://books.google.com.au/books?id=OEIQAQAAIAAJ.

- - . 2001. Sex, Ecology, Spirituality: The Spirit of Evolution, Second Edition. Shambhala. https://books.google.com.au/books?id=U5UJUChbuioC.

- - - 2007. Integral Spirituality: A Startling New Role for Religion in the Modern and Postmodern World. Shambhala. https://books.google.com.au/books?id=n-92sivPE2sC. 